\title{
ON FORCED RESPONSE OF A ROTATING INTEGRALLY BLADED DISK: PREDICTIONS AND EXPERIMENTS
}

\author{
Claude Gibert \\ Laboratory of Tribology and System Dynamics \\ UMR CNRS 5513, Ecole Centrale de Lyon \\ 36 av. Guy de Collongue, 69134 Ecully Cedex, France \\ claude.gibert@ec-lyon.fr \\ Fabrice Thouverez \\ Laboratory of Tribology and System Dynamics \\ UMR CNRS 5513, Ecole Centrale de Lyon \\ 36 av. Guy de Collongue, 69134 Ecully Cedex, France \\ fabrice.thouverez@ec-lyon.fr
}

\author{
NOMENCLATURE \\ BW - backward travelling wave \\ FRF - frequency response function \\ $\mathrm{FW}$ - forward travelling wave \\ EO - engine order \\ LDV - laser Doppler vibrometer \\ $\mathrm{N}$ - number of sectors in the blisk \\ PZT - lead zirconate titanate piezoelectric actuator \\ SW - standing wave \\ TW - travelling wave \\ 4ND-3 - third mode of four nodal diameters \\ $\Omega$ - rotor rotational frequency, $\mathrm{rpm}$ \\ $\sigma-$ standard deviation of random distribution \\ $\omega$ - excitation frequency, $\mathrm{Hz}$
}

\section{ABSTRACT}

An experimental setup is described which permits to rotate a bladed disk in vacuum and to measure its dynamic response to excitations provided by some embedded piezoelectric actuators.

A particular spatial placement of actuators associated with phase-shifting electronic circuits is set for simulating travelling wave excitations with respect to the rotating frame. The system is demonstrated on an actual high-pressure compressor (HCP) integrally bladed disk. The dynamic response of the blisk is analyzed experimentally and results are correlated with those

\author{
Vsevolod Kharyton * \\ Laboratory of Tribology and System Dynamics \\ UMR CNRS 5513, Ecole Centrale de Lyon \\ 36 av. Guy de Collongue, 69134 Ecully Cedex, France \\ vsevolod.kharyton@ec-lyon.fr \\ Pierrick Jean \\ Snecma, SAFRAN group \\ Site de Villaroche, Moissy Cramayel 77550, France \\ pierrick.jean@snecma.fr
}

obtained from a simplified finite elements model taking into account Coriolis effect.

The paper focuses on the influence of the latter which is most of the time neglected and its implication on the forced response levels is studied into two situations without or with mistuning.

Key words: bladed disk, forced response, Coriolis effect, travelling wave response, mistuning

\section{INTRODUCTION}

High cycle fatigue (HCF) is caused by the excitations applied to rotating parts of gas-turbine engine as they are exposed to stationary disturbances in the flow field. This excitation is often called engine order excitations, depending on the number of equally spaced obstacles present either upstream or downstream of the bladed disk. On the other hand, modern technology have leaded to integrally bladed disks (blisks) with complex three-dimensional designs, which feature a very weak internal damping and can lead to a risk of HCF. Numerical methods are able to take into account the different phenomena inherent to these kinds of systems, namely mistuning, stress stiffening, spin softening and Coriolis effects. All of them are useful to predict precisely the resonant conditions at operating rotation frequency. Nevertheless, the Coriolis effect is less addressed in literature, both numerically and experimentally. For example, Coriolis effects and their mutual interaction with mistuning is studied in [12] numerically with a lumped mass model and experimentally by means of a

*Author for correspondence 
specially designed so-called swept test piece with 24 blades having the sweep angle of $15 \mathrm{deg}$. allowing a strong Coriolis coupling of blades vibrations. In [5], the effects of the Coriolis force and the magnitude of disorder on the localization phenomenon of a rotating bladed-disk system were investigated numerically. Obtained results indicate that the Coriolis force may enhance the localization phenomenon. The gyroscopic effect is taken into account in [6] by considering a flexible bladed disk-shaft assembly, where it is shown that 0 and 1 nodal diameters (ND) modes are affected by the coupling. As testing is also necessary for studying the dynamics of blisks, different experimental systems have been reported in the literature. Travelling wave excitation systems have been designed for testing blisk-like (academic) or real structures using piezoelectric, acoustic or magnetic actuators applied to stationary bladed disks. Distinction can be made between some of them named "rotating" and others, so-called "phase shifting" using a stationary disk. Rotating configuration concerns to any rig in which either the excitation source or the test piece is rotated to create the travelling wave. With phase shifting configurations the excitation sources remain stationary, but differ from sector to sector by a constant intersector phase angle simulating engine order (EO) excitation. In [9], a stationary 12-bladed disk with simple geometry is subjected to a travelling-wave out-of-plane "engine order" excitation delivered via phased piezoelectric actuators mounted on the blades. In [8], a testing system using either electro-magnetic or acoustic excitation on a stationary 18 -blades blisk is presented. In [13], travelling wave excitations are delivered to a stationary real 24-bladed disk acoustically by small phased-speakers mounted behind each blade. Some rotating systems are reported in [8] which use permanent magnets or air impingement for providing phased pulses rather than harmonically pure phased sinusoid as with phase shifting devices. This type of excitation is said to be more representative regarding to true forces meet in a gas-turbine engine consisting in a fundamental harmonic, as well as lower amplitude ones at integer multiples of a fundamental frequency. Although classified as rotating, most of the above cited systems work with a stationary disk. The presented test rig consists of a shaft where any test piece can be adapted, rotated in vacuum and excited by embedded piezoelectric actuators. The use of vacuum prevents from generating aerodynamic excitations and allows characterizing the damping of the structure itself. This rig has been already used for several kinds of experimental tests mainly involving damping characterization. Indeed, the ability to excite at any frequency and at arbitrary level, at a given rotating speed in vacuum allows to carry out precise estimation of damping effect [4],[11],[14] using different test configurations.

The primary focus of the paper is to show the influence of Coriolis forces on dynamics of realistic industrial bladed disks. Normally, during turbomachinery components analyses its effect is supposed negligible, whereas influence of centrifugal forces is integrated to nowadays calculations.
The paper is organized as follows. First, the experimental setup is described focusing on the travelling/standing wave excitation system and followed by the derived test data. Then the simplified model of the bladed disk is presented to perform dynamic characterization of the disk and to examine Coriolis forces influence on the disk dynamics. Also a range of problems concerning the forced response on the excitation, which is fixed with regard to the disk, is discussed. Special attention is paid to representation of the excitation system within the bladed disk model. Finally, the questions of mistuning influence on the disk dynamics in conjunction with Coriolis effect are conversed. The main purpose of the part dedicated to numerical simulations is the comparison with the experimental results and the ability to continue numerically investigation of a wider range of dynamic parameters influence.

\section{EXPERIMENTAL SET UP}

\subsection{General Description}

A general schema of the test rig is shown in Fig. 1. The system consists of a shaft supported by precision preloaded ball-bearings and driven by an electric motor. A test piece can be adapted to the shaft by means of a stiff supporting disk rotating in a vacuum tank. Herein, a 36 blades blisk of HCP stage is investigated. Excitation is provided by embedded piezoelectric actuators which are driven by power amplifiers through an end-shaft slip-ring. Measurements are taken by means of strain gauges and transmitted using an inductive telemetry system.

The use of vacuum (less than 10 mbars) permits to avoid any effect coming form surrounding air. The excitation signal can be set in term of frequency, spatial distribution and strength independently from the rotating speed. The test pieces are not generally run at designed speed within this laboratory rig, though important phenomena can be observed: effect of the centrifugal loading on vibration with friction interfaces, stress stiffening and Coriolis effect.

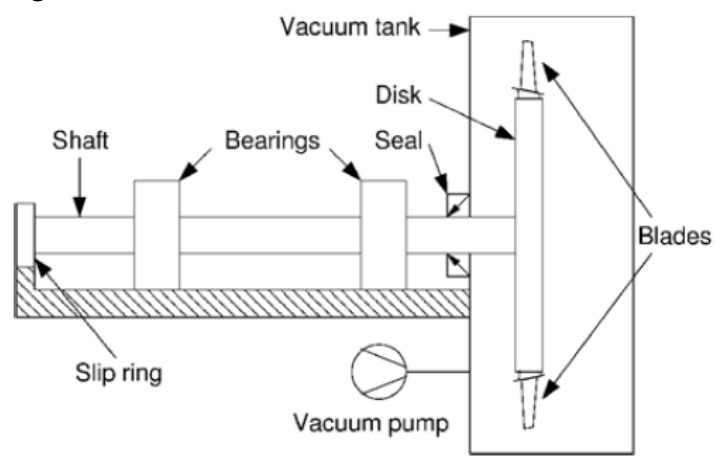

Fig. 1 Test rig scheme ([4])

\subsection{Test Rig Excitation System}

The used excitation system is partially described in [11], where non-rotating excitation with respect to the blisk is discussed. The novelty brought by current study concerns the generation of a travelling wave excitation. The actuators are 
lead zirconate titanate (PZT) piezoelectric plates bonded on the blisk (Fig. 2a).

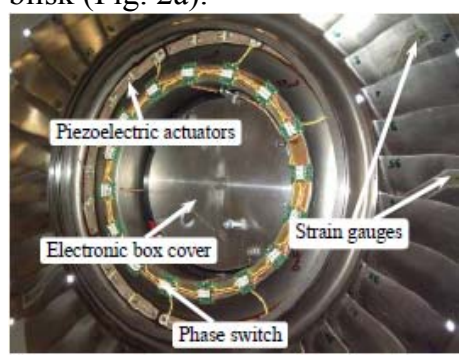

a

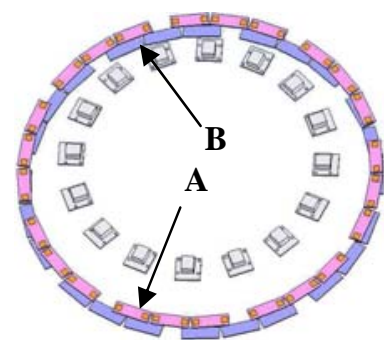

b
Fig. 2 Instrumented blisk[11]: a - instrumentation,

$\mathrm{b}-$ Position of the two series PZTs on faces A and B

The choice for PZT locations was motivated by several considerations. Compared to other ones, relatively high strain was predicted on the disk on the computed mode shapes of interest showing a good potential for their excitation and locally plane surfaces were well suited for bonding of PZT plates. Twenty-four PZT plates are positioned circumferentially on the blisk on both front (A) and rear (B) faces as seen in Fig. $2 \mathrm{~b}$. Actuators are gathered into 8 separated groups of 3 PZTs. All PZTs within a group are connected in parallel and will transmit in phase stresses for a given voltage at their electrodes

The electrical connections of the groups are shown in Fig. 3. A signal generator gives a common signal $v(t)=V \cos \omega t$ to the amplifiers, one of them (amplifier 2 in Fig. 3) being driven through a $\pm \pi / 2$ phase-shifter circuit. The output power of each amplifier is passed through the slip-ring to two series of PZT groups on the rotating blisk.

Sixteen small and light-weight electronic voltage inverters using DIP switches $\left(\mathrm{SW}_{\mathrm{Ai}}, \mathrm{SW}_{\mathrm{Bi}}, i=1 \ldots 8\right.$, as referred in Fig. 3) are assembled on printed circuit boards. Each group has its own inverter allowing setting its phase to 0 or $180 \mathrm{deg}$. relative to $v(t)$. These are gathered on the front side and bonded on the bottom massive part of the blisk section allowing the user to set any nodal diameter excitations. Each face is able to produce a standing wave excitation with nodal diameter numbers $0,1,2$ or 4 depending on the switches settings. For example, inverting groups 1, 3, 5, 7 regarding to groups $2,4,6,8$ will results in a $4 \mathrm{ND}$ excitation (if groups are numbered sequentially around the circumference of the blisk).

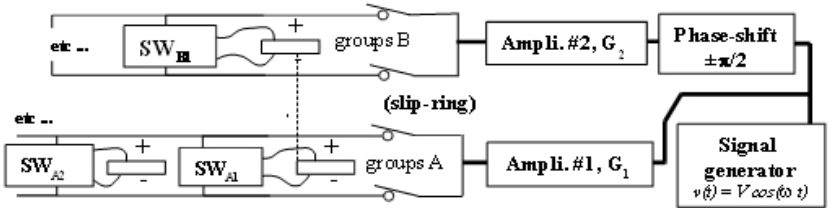

Fig. 3 Electrical connections of actuators for TW excitation

The amplifiers gains $\left(\mathrm{G}_{1}, \mathrm{G}_{2}\right)$ can be adjusted for calibration purpose. As detailed after, a four nodal diameters travelling wave excitations can be provided as far as the faces A \& B of the blisk are similarly equipped with 8 groups of actuators spatially shifted from by an angle of $22.5 \mathrm{deg}$. $(\pi / 8)$ along the circumference (Fig. 2b). The travelling wave will result in the combination of the excitations coming from face $\mathrm{A}$ and face B together spatially and temporally shifted. Note, that switches settings with nodal diameter 1 and 2 will not result in a pure diametric travelling wave excitation as the spatial phase shift of PZTs was designed previously for studying 4ND modes [11] (a standing wave component would be present in that case).

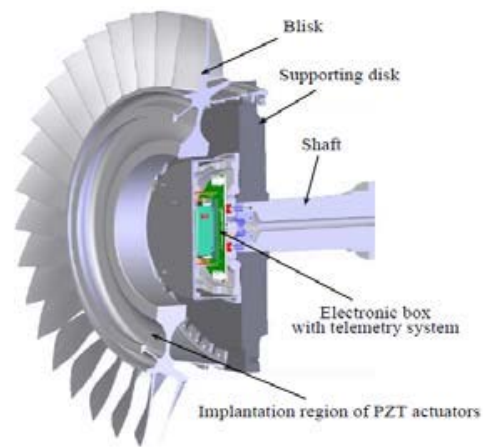

Fig. 4 Integration of blisk and telemetry [11]

An improvement of the system can be done in order to have the possibility for any spatial shift angle $\pi / 2, \pi / 4, \pi / 6 \ldots$ between face $\mathrm{A} \& \mathrm{~B}$ for respectively $\mathrm{ND}=1,2,3 \ldots$, using a more complex arrangement of PZT plates and switches.

Compared to some of the literature, one drawback of the system is that the force can not be directly measured and the system is not easily applicable from one blisk to another as in [7], [8]. The advantage is that the frequency content and the magnitude of the voltage can be adjusted accurately. The system is rather simple as it uses only one phase-shifter circuit, two amplifiers and a basic circuitry and it was designed for relatively strong excitation levels in order to study the influence of friction damping devices [11] .Given the high quality factor of the blisk itself, a lower level would have been sufficient for a study limited to modal or mistuning characterization.

\section{EXPERIMENTAL RESULTS}

\subsection{Measurements}

The dynamic response of the rotating blisk is measured by means of 12 semiconductor strain gauges. Eight of them are cemented on the suction side of blades (Fig. 2), while 4 others are on the disk (not shown). Thanks to their small size and high sensitivity, they are well suited for measurement at high frequency without significant perturbation of the test piece. 8channels telemetry system has been integrated for signal conditioning, anti-aliasing filtering, $\mathrm{AD}$ conversion close to the sensors minimizing wire length. Digital output of the 8 sensor modules are multiplexed and transmitted to the receiver in the laboratory frame using an inductive coil mounted on the rear end of the shaft. The conditioning electronic cards are assembled on a main board and the whole carefully maintained inside of a metallic box fixed on the supporting disk. Special attention was paid to separation of low and high voltage circuits and shielding and to the use of twisted pairs in order to minimize electrical perturbation of measurements by the 
excitation system. Piezoelectric precision voltage drivers (i.e. amplifiers) provide low-voltage representations of the highvoltage output used as the reference signal for some FRF measurement. It has been checked that the response of the structure is linear regarding to this reference. Classical sensors, such as accelerometers, pressure and temperature gauges, and tachometer are also used.

\subsection{Excitation characteristics}

\subsubsection{A Diameter Excitation}

The harmonic content of the proposed excitation method will be analyzed by means of simple models as shown in Fig. 5 and Fig. 6. considering the Fourier analysis of four nodal diameter (4ND) square signals. These idealized signals intend to represent the distribution of the excitation provided by PZTs to the disk along its circumference. The patterns in Fig. 5a and Fig. 6a are square signals, the second one being slightly different because of some zero amplitude zones. These zones correspond to the spaces left between each group of 3 PZTs in Fig. 2b. Amplitude spectrums are given in Fig. 5b and Fig. $6 \mathrm{~b}$.

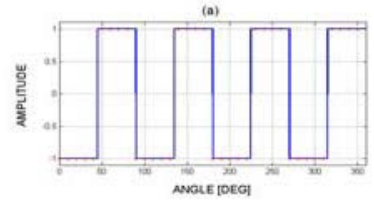

(c)

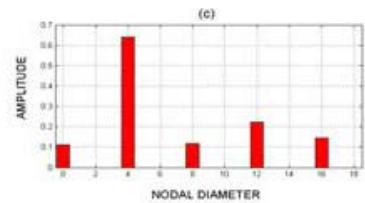

Fig. 5 Analysis of a 4ND excitation: square signal
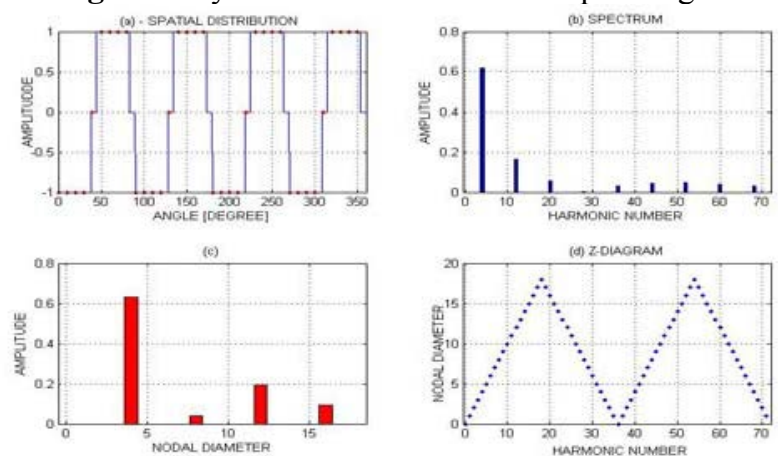

Fig. 6 Analysis of 4ND excitation: squares spaced signal

In both case, we have fundamental (with number $N_{h}=4$ ) with odd harmonics i.e. $N_{h}=12,20,28,36,44 \ldots$. The higher harmonics are the same but amplitudes are different for the two patterns. Note that the envelope of the spectrum decays as $1 / N_{h}$. The so called ND-spectrum diagrams (Fig. 5c and Fig. 6c) give the corresponding amplitude of the discrete Fourier transformation (DFT) with $N$ points over the circumference. The Z-diagrams (Fig. 5d and Fig. 6d) of the blisk with 36 blades indicate a harmonic participation. For example, the harmonics $\mathrm{N}_{\mathrm{h}}=4,32,40,68,76 \ldots$ will excite a $4 \mathrm{ND}$ mode.
Interestingly, the influence of the zero zones is seen in the Fig. $6 \mathrm{c}$ where the participation to the OND modes vanishes. This effect has been confirmed by means of the FE blisk model simulating the forced response to the 4ND excitation distribution as discussed bellow (section 4.3).

\subsubsection{Uniformity of Excitation}

The effect of non-uniformity of force distribution on the harmonic content will be discussed in this section. Nonuniformity can be caused by errors in positioning, phase or strength of actuators [8]. The effect on variations in strength among the different groups of actuators is estimated by analyzing a perturbed square spaced signal as shown in Fig. 7
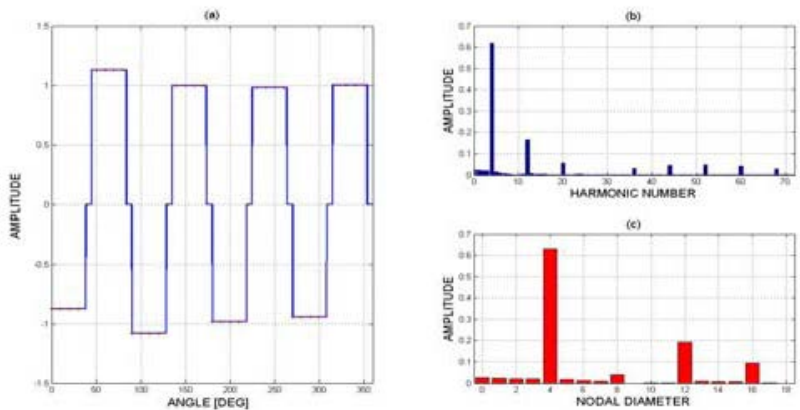

Fig. 7 Analysis of the perturbed signal

In our case, the strength of forces depends on the thickness of bonding layers as mentioned in [9] and it is believed to be the main possible source of non-uniformity. Despite this perturbation, the results, in terms of excited diameters, are not very different from the unperturbed case (Fig. 6). One can see that additional low ND appear weakly excited, resulting from perturbations in amplitude of $\sim 25 \%$.

The second investigation about the non-uniformity of the excitation is performed experimentally. Eight FRFs measured at blade 2 by means of strain gauges using successively PZTs groups 1 to 8 are shown Fig. 8. The forced response is measured near the resonance of the 4ND-3 mode which mode shape will be presented bellow and can be considered as a socalled extended mode shape [8] (i.e. not localized one).
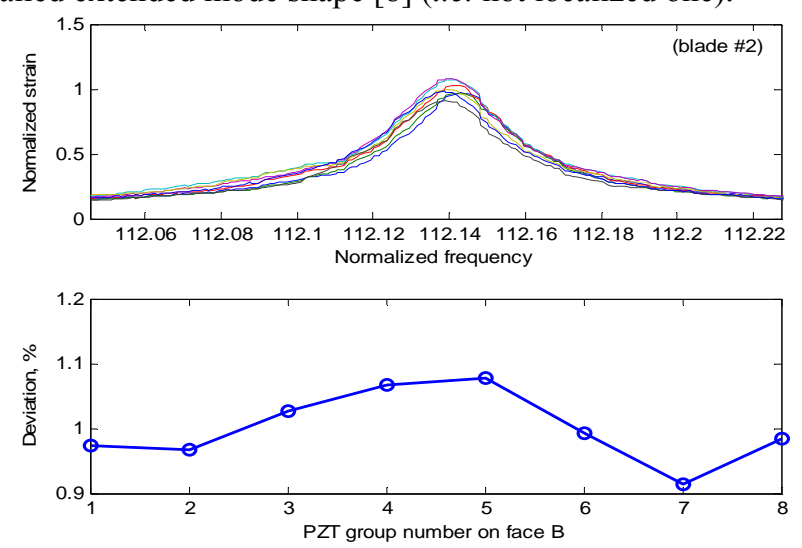

Fig. 8 Experimental test of uniformity of PZT excitation 
The maximum deviation observed from a mean value between amplitudes at peaks is of the order of $10 \%$ that can be considered as a very satisfactory result. Consequently, it is believed that the excitation is practically uniform. The test has been performed for both faces (A \& B) of actuators

\subsubsection{Response to a Diametric Excitation}

The FRFs measured at one blade is shown in Fig. 9 (bottom) with the blisk excited by the 4ND excitation in a frequency range containing modes with different NDs than the excitation (some of them are indicated). A single face excitation (i.e. only PZTs located on one face) is used with the blisk at rest in order to perform this check in the simplest condition (the test has been performed separately with the two faces). Satisfactorily, the response to the 4ND excitation is dominated by the response of the 4ND modes. Although, as referred in [8], distortions of modes caused by mistuning specially for higher NDs (see frequencies between 95 and 100 here), and non uniformity of the excitation (as shown in 3.2.2), can explain that modes with other NDs are present in the FRF. Further, a comparison can be made with the 8 FRFs obtained using again the 8 groups of PZTs separately which are presented in Fig. 9 (top).
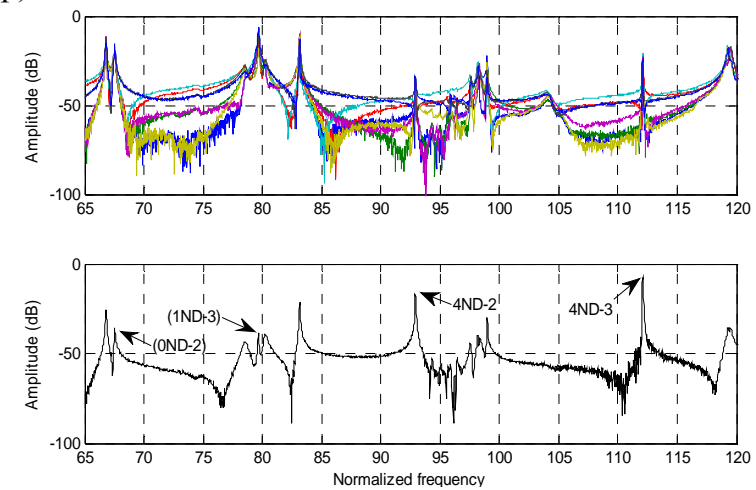

Fig. 9 Response to 4ND standing wave excitation (bottom) and to individual groups of PZT (top)

Comparatively, the maximum amplitude of response of the 4ND modes are raised $(\sim 18 \mathrm{~dB})$ using the $4 \mathrm{ND}$ excitation, and other ones with different NDs are lowered (-10dB to $-25 \mathrm{~dB})$. This occurs in particular for the 0ND mode at frequency $\sim 67$, for which all FRFs in Fig. 9-top have the same amplitude, 20dB higher than this obtained in Fig. 9-bottom.

This test shows the quality of the 4ND excitation in its ability to excite predominantly the modes of the same ND.

\subsubsection{Travelling Wave Excitation \\ General engine order excitations}

Rewriting from [3], the assumed periodic excitation seen by an individual reference sector (say the number 0 ) rotating downstream of $n$ stator blades at a rotating speed $\Omega$ can be expressed by a series of harmonic components

$$
f_{0}(t)=\sum_{p} a_{p} \cos (n p \Omega t)+b_{p} \sin (n p \Omega t)=\sum_{p} C_{p} \cos \left(n p \Omega t+\varphi_{p}\right)
$$

where $\mathrm{C}_{p}$ and $\varphi_{p}$ can be expressed in terms of $a_{p}$ and $b_{p}$, which are the Fourier series coefficients of the periodic function.

As a result, the excitation seen by the $j$-th of the $\mathrm{N}$ sectors will be (assuming the blades are numbered in the rotating direction)

$$
f_{j}(t)=\sum_{p} C_{p} \cos \left(n p\left(\Omega_{r} t-j \frac{2 \pi}{N}\right)+\varphi_{p}\right)
$$

The excitation is seen as a superposition of several travelling wave components with $n p$ and frequencies equal to $n p \Omega$. Note, that the propagation speed of these travelling waves components regarding to the blisk is $\Omega$. The direction of propagation in the rotating frame of reference is reversed to the direction of rotation.

\section{Test rig signal generation}

In the rig, a $m$-nodal diameter standing wave excitation provided by the actuators of the face A, driven by a sine wave at the frequency $\omega$, is assumed to be of the form:

$$
p_{A}(t, \theta, r)=V \cos \omega t\left(\sum_{k=0,1, \ldots} d_{k} \cos (2 k+1) m \theta\right) u(r)=v(t) p_{A}^{0}(\theta, r)
$$

where $r$ is the radial coordinate, $u(r)$ is the function describing the radial distribution of excitation transmitted to the blisk, $d_{k}$ are the Fourier series coefficients. This form is justified because the PZT groups of the face are driven by a common harmonic signal and only odd spatial harmonics are present as found in 3.2.1. Also, as far as any nonlinearity is brought to the structure (as in [11]), the response of has been shown to be proportional to the voltage $v(t)$, which is chosen as the reference for FRF measurement.

Assuming the same for face B with only difference in the temporal phase shift of $90 \mathrm{deg}$. and spatial shift $\pi /(2 \mathrm{~m})$, we have:

$$
p_{B}(t, \theta, r)=V \sin \omega t\left(\sum_{k=0,1, \ldots .} d_{k} \sin (2 k+1) m \theta\right) u(r)
$$

Then, if faces A \& B are acting in the same time, the resulting signal will be:

$$
p(t, \theta, r)=p_{A} \pm p_{B}=V\left(\sum_{k=0,1, \ldots} d_{k} \cos (\omega t \pm(2 k+1) m \theta) u(r)\right.
$$

Note that the coefficients $d_{k}$ are assumed to be the same in both expressions for $p_{A}$ and $p_{B}$. This will be ensured by the calibration process discussed later. The resulting signal can be interpreted as superposition of travelling waves with different nodal diameters $(m, 3 m, 5 m, \ldots)$, but with the same frequency $\omega$. Therefore each term represents a travelling wave propagating at different speeds $\omega /(2 k+1) / m$, which makes a qualitative difference with the expression of $f_{j}(t)$ above. The 
direction of propagation depends on the chosen sign (+ or - ), independently from the actual rotating direction of the blisk.

As far as typical signals show that harmonics amplitudes are decaying fastly with their number, it is believed that the produced excitation is mainly composed of the fundamental one $(m)$. For instance, from Z-diagram, a 4-nodal diameter mode could be excited by harmonics $32,40,68,76, \ldots$, but these contributions are very small. Also, note that the propagating speed is much higher than the rotating speed of the rig.

Although, as the rig is rotating much slower than the actual HPC, the propagating speed in the rig should be realistic. On the contrary, other effects depending on the rotating speed such as frequency shift induced by stress stiffening and Coriolis forces will probably be not in the corresponding importance. Nevertheless, it is rather intended to produce typical effects than to simulate real conditions by means of the rig.

\section{Calibration procedure}

Calibration of phase and amplitude is inherent to any kind of travelling wave excitation systems in order to take into account the non-uniformity and positioning errors. In [9], the gain of amplifiers is adjusted to account for variations in the strength of the bonding of actuators to the blade roots. It is carried out by considering the peak amplitude to single blade excitation. In this way, the authors could make each actuator to provide the same excitation amplitude for all blades. In [13], the speakers are driven by an array of phase-synchronized signal generators. The excitation signals are calibrated so that all speakers provide the same strength excitation at any frequency, but each successive speaker receives a signal with a specific phase lag behind its neighbor.

As indicated above, the travelling wave is generated by a combination of excitations provided by actuators located on two faces (A \& B) and in order to succeed we should simulate forces of the same magnitude.

This is the objective of the calibration process illustrated in the case of the 4ND excitation. The following operations are performed. First, a given level of output voltage $V$ for the signal generator is chosen. Then, FRFs are obtained from measurements by LDV at the 36 blades in the vicinity of the natural frequency of a four nodal diameter pair of modes (the 4ND-3 at frequency 112 ) with each one of the two actuator series (A \& B) in turn and the blisk being at rest.

The FRFs are analyzed by means of modal analysis using the following form (it is written for excitation at the face A):

$$
h_{j}^{A}(\omega)=\frac{u_{j}^{A}}{V}(\omega)=\frac{\psi_{c}^{j}{ }^{T} \psi_{c} p_{A}^{0}}{\omega_{c}^{2}-\omega^{2}+2 i \xi_{c} \omega_{c} \omega}+\frac{\psi_{s}^{j}{ }^{T} \psi_{s} p_{A}^{0}}{\omega_{s}^{2}-\omega^{2}+2 i \xi_{s} \omega_{s} \omega}
$$

where $u_{j}$ is the displacement response at blade $j(j=1, \ldots, 36), \psi_{\mathrm{c}}$ and $\psi_{\mathrm{s}}$ are the two mode shapes of pair with their associated frequencies $\left(\omega_{c}, \omega_{s}\right)$ and damping ratios $\left(\xi_{c}, \xi_{s}\right)$. Usual terms taking into account extra-band modes could have been neglected.

The nominators in the expression of the FRFs will be called the modal residuals, which are real vectors and will be written $\mathrm{R}_{\mathrm{cA}}, \mathrm{R}_{\mathrm{sA}}, \mathrm{R}_{\mathrm{cB}}, \mathrm{R}_{\mathrm{sR}}$, for instance:

$$
R_{c A}^{j}=\psi_{c}^{j T} \psi_{c} p_{A}^{0}, R_{s A}^{j}=\psi_{s}^{j T} \psi_{s} p_{A}^{0}, \quad j=(1, \ldots, 36)
$$

Two splitted modes are found in practice having slightly different frequencies and nearly the same damping ratio. Fig. 10 and Fig. 11 show the so-called modal residuals extracted from FRFs in the two cases with actuators A and B (blades equipped with strain gauges are represented in white).
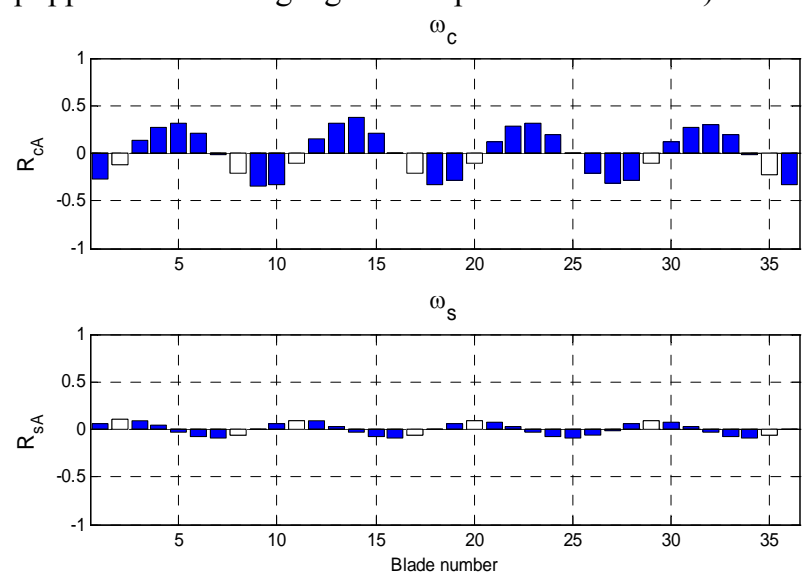

Fig. 10 Modal residuals (4ND excitation with face A) $\omega_{c}$
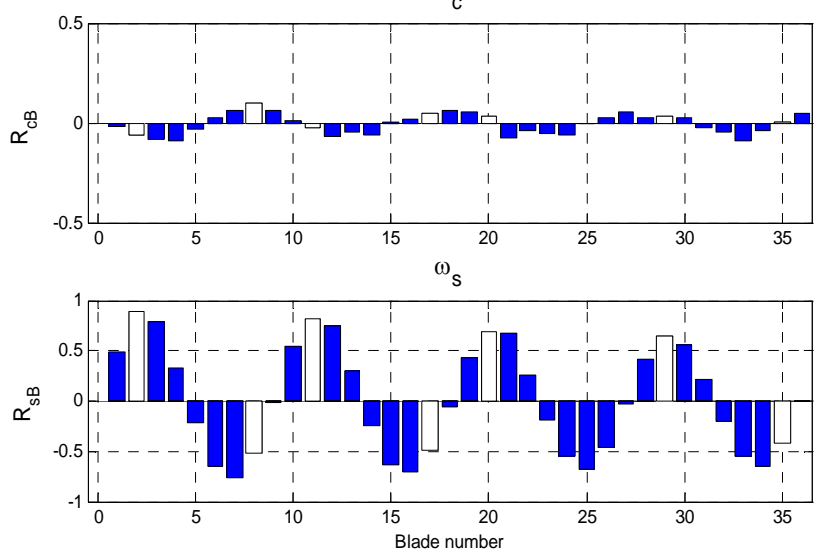

Fig. 11 Modal residuals (4ND excitation with face B)

It was observed that the actuators of face A excite predominantly the first one of the doubled mode while the actuators of face $\mathrm{B}$ excite the second one with an upper natural frequency (we have $\omega_{c}<\omega_{s}$ ). The amplitude of residuals, obtained with the face B is nearly two times higher. This indicates a higher generalized force with the face $\mathrm{B}$ for the same common voltage amplitude of reference $(V)$. This difference is attributed to the thickness of the bonding layer which has been optimized for the face B. Also a finite elements analysis of the strain distribution can give some part of the 
explanation as the strains are slightly different at the two actuators locations for the same mode shape. At the end, the initial gain of the amplifier 1 is updated giving the ratio between two identified modal residuals $\left(\mathrm{R}_{\mathrm{sB}} / \mathrm{R}_{\mathrm{cA}} \approx 2.3\right)$ in order to calibrate the excitation: $\mathrm{G}_{1}{ }^{\text {updated }}=2.3 \times \mathrm{G}_{1}{ }^{\text {initial }}$.

\section{Validation of the travelling wave excitation}

In this section, the idea is to validate the travelling wave excitation indirectly through its effect on the response of the blisk. But, as it will be shown after, travelling wave response can be obtained even with a standing wave excitation, if the blisk is rotating due to Coriolis effect. Then, for clarity the validation will be performed with the blisk at the rest. Although, even in that case and because of the mistuning, a care have to be taken in choosing the excitation frequency $(\omega)$ used to demonstrate the nature (SW or TW) of the response. In that way, several cases are considered whether the blisk is stationary or at rest and the excitation is a standing or a travelling wave.

Case 1: Stationary disk - standing wave excitation

In this case, the blisk is non-rotating and excited by actuators of face A. Fig. 12 shows the FRFs measured at all blades with LDV at resonances of the pair of modes 4ND-3.

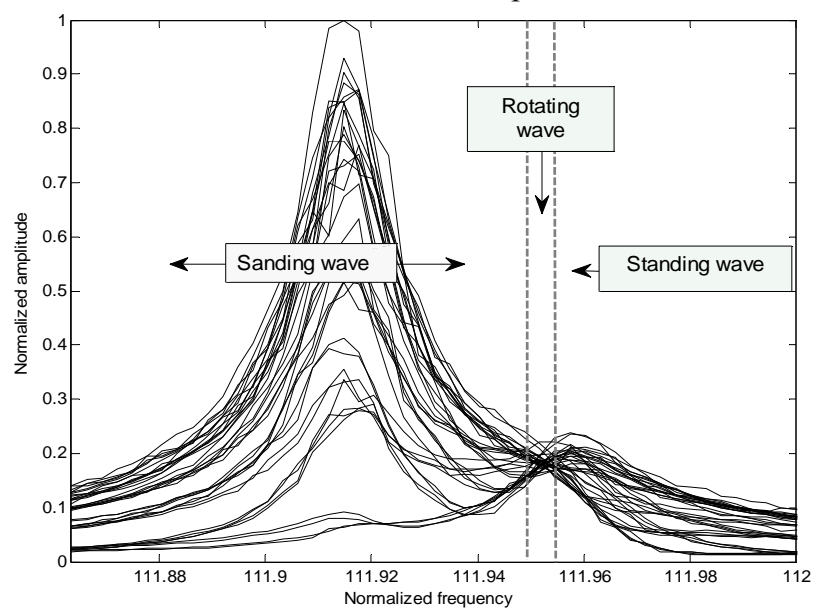

Fig. 12 FRFs measured at all blades, excitation with face A

As said before, two very close peaks are visible with the first one responding mostly. Then, the shape of the forced response is examined at some particular frequencies (Fig. 13 and Fig. 14).

At a frequency above the resonances (Fig. 13), it is possible to recognize a 4ND mode looking at the modulus of amplitudes and trivial phases of 0 or $180 \mathrm{deg}$ between blades which characterizes a standing wave of response. Secondly, when the frequency is chosen near the second resonance (Fig. 14), the quality of the response is quite different since amplitudes of all blades are practically of the same and the phase between two consecutive blades has a constant value of $40 \mathrm{deg}$. The phase of blade $j$ relative to blade $i$ is given by (in deg)

$$
\varphi_{i, j}=360 m / N(i-j)
$$

For a wave with $m$ nodal diameters travelling from blade $i$ to $j$, a negative phase value indicates travelling wave response propagating in the direction of increasing blade numbers. FRFs are normalized by the maximum over all blades.

The modal analysis of FRFs indicates that at frequency near 111.95, the modal participations of the two spatially orthogonal modes have the same magnitude and a relative phase of nearly $90 \mathrm{deg}$. since the second mode is near its resonance. Apart that particular situation resulting in a TW, the response is dominated by the standing wave mode shape for frequency bellow or above 111.95 which therefore reflects the nature of the excitation unambiguously and will be used to validate the TW excitation.
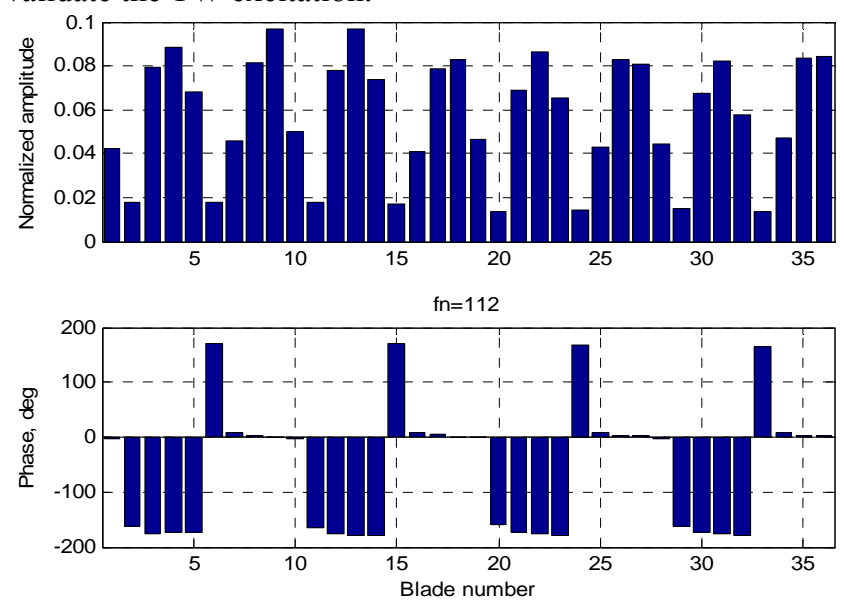

Fig. 13 Standing wave response to standing wave excitation (frequency $\sim 112$ )
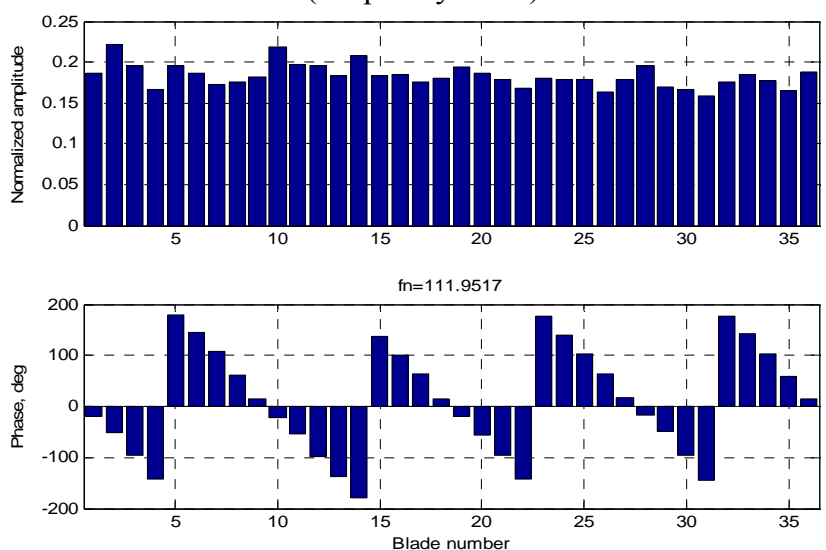

Fig. 14 Travelling wave response to standing wave excitation (frequency $\sim 111.95$ )

Case 2: Stationary disk - travelling wave excitation

The response to the travelling wave excitation at a forcing frequency producing the standing wave response (as in case 1 for a frequency above 111.95) is illustrated in Fig. 15 and it shows a travelling wave. Blades instrumented with a strain gauge are indicated in white. Note that the relative phase between these blades is 0 or 120 deg., and that it would have been 0 or $180 \mathrm{deg}$. with a standing wave response. 

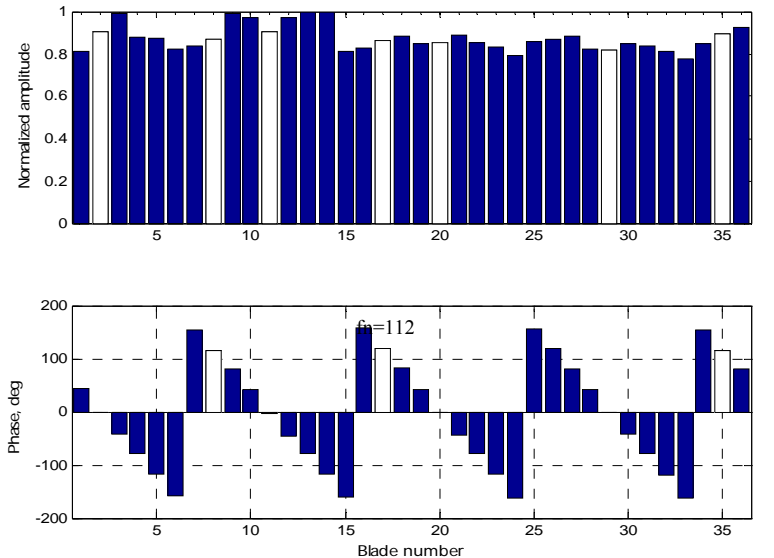

Fig. 15 Travelling wave response (all blades with LDV) to travelling wave excitation (frequency $~ 112$ )

\subsubsection{Strain gauge response}

As far as the disk will be rotated, only embedded strain gauges will give some information about the nature of the forced response of the blisk. Therefore, we need to identify the mode shape using only 8 gauges in the case of a travelling wave response. This is done by characterizing confidently the mode shape at rest by means of extended measurements with LDV at all blades and looking at the gauge in the same experiment. This is illustrated in Fig. 16, where amplitudes and inter-blade phases are measured with gauges. The excitation signal of the amplifier 2 has been inverted (set with $-\pi / 2$ instead of $+\pi / 2$ used in Fig. 15) in order to illustrate in the same time the ability to change the direction of propagation of the wave. One should then compare the phase diagrams of Fig. 16 and of Fig. 15 for blades 2, 8, 11, 17, 20, 29, 30. The found values of 0 deg. and \pm 120 deg. depending on the blade number validate that we have 4ND travelling waves (TW) with different directions of propagation (FW or BW). For instance, we have a relative phase $\varphi_{8,11}=-120$ deg between blades 8 and 11 in the Fig. 15 while $\varphi_{8,11}=+120$ deg in Fig. 16 . Note that the phase of $+80 \mathrm{deg}$. of the strain gauge located on the platform, near the blade 22 can be compared to the phase of $-80 \mathrm{deg}$. measured by LDV at the tip of this blade in Fig. 15.
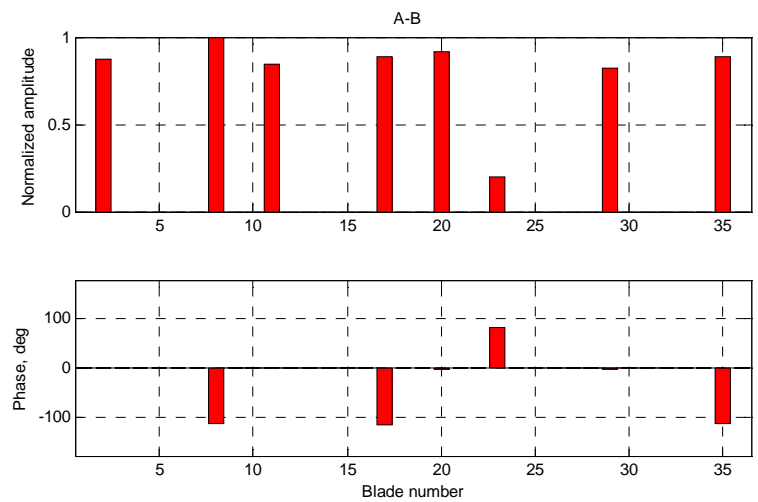

Fig. 16 Travelling wave response measured by 8 strain gauges
Note, that all amplitudes are nearly the same except for the stain gauge located on the platform (close to blade 22) which is of lower response. Also, one could see that looking only at amplitudes of strain gauges responses may not be always sufficient for identifying clearly a SW or a TW depending on the distribution of the sensors on the SW mode shape as with the one shown in Fig. 16 (see the shape of $R_{s B}$ ).

From this section, the excitation system is validated in its ability to produce a ND excitation forward or backward. Also, it has been shown that the nature of the response can be identified clearly in the case of a rotating disk using the gauges signals.

\subsection{Response of Rotating Blisk}

The dynamic behavior of the rotating blisk is studied in this section. Two cases are considered.

Case 3: Rotating disk - standing wave excitation

Fig. 17 shows different FRFs obtained at the blade 2 in the vicinity of the $4 \mathrm{ND}-3$ mode at different rotating speeds from 0 to $2500 \mathrm{rpm}$ (in vacuum).

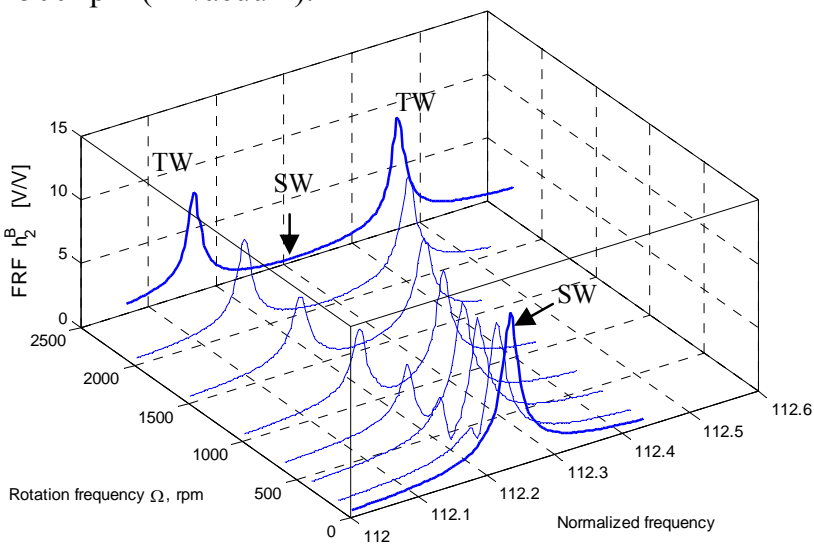

Fig. 17 FRFs at mode 4ND-3 with excitation on the face B

The standing wave 4ND excitation is provided by actuators of the face B. A splitting of the single peak appears and increases with the rotation speed. The modal analysis using all strain gauges revealed two complex mode shapes corresponding to a $\mathrm{BW}$ and a $\mathrm{FW}$ travelling waves and a real mode shape at rest. Phase analysis of forced response shows SW response between the two peaks for the rotating disk due to superposition of two TW modes participation.

Similar responses have been obtained for the 2ND-2 mode $\left(2^{\text {nd }}\right.$ mode with two nodal diameters) which frequencies are analyzed in the section 4.2.

Case 4: Rotating disk - travelling wave excitation

The response of the rotating blisk to the traveling wave excitation is illustrated in Fig. 18. It appears to be dominated by one peak (at frequency $\sim 112.41$ ) at which vicinity it has been identified as the travelling wave (by means of phases in accordance with the discussion in section 3.2.5). If the direction of the TW excitation is changed, symmetric situation (not shown here) occurs with the main peak at frequency $\sim 112.15$ 
that corresponds to the travelling wave response propagating in the opposite direction. The response at $0 \mathrm{rpm}$ (for the same TW excitation) is superimposed on the diagram for comparison. This latter is composed of superposition of two real modes found bellow (Fig. 10 and Fig. 11) with modal amplitudes of same modulus as the excitation has been calibrated (see 3.2.4). Because of mistuning, two peaks appear. The resulting response is identified as of standing wave at the resonances.

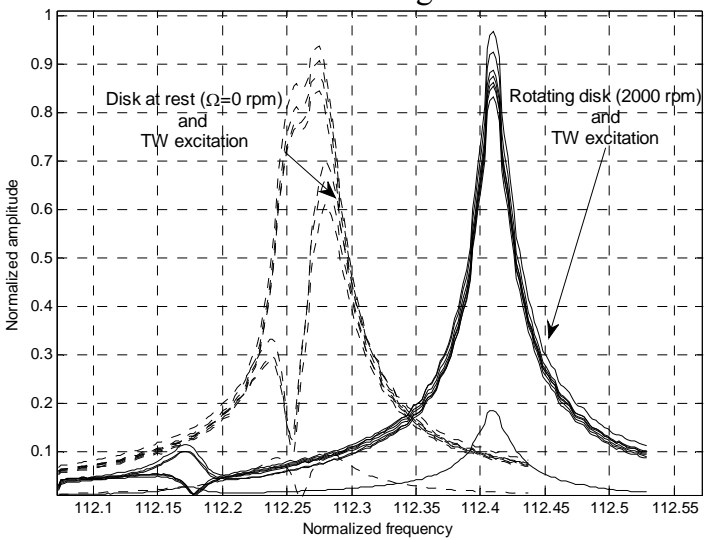

Fig. 18 Forced response to the TW excitation without and with Coriolis effect: strain gauges FRFs at 0 and $2000 \mathrm{rpm}$

Therefore, the difference found in Fig. 18 between the response of the blisk with Coriolis effect (at $2000 \mathrm{rpm}$ ) and without it (at $0 \mathrm{rpm}$ ) concerns the resonance frequency and the distribution of maximum amplitudes over the different blades.

\section{NUMERICAL MODEL}

\subsection{Free Vibration Characteristics of Bladed Disk}

The numerical section of the paper focuses on simulation of the bladed disk dynamics. As the disk has rather complicated geometry it was decided to accomplish all following calculations using a simplified full model which allows applying some mistuning effect. Finite elements modeling was performed using ANSYS [1].

In order to adjust the simplified model the comparison with the realistic finite elements model was done (Fig. 19). The model contains CAD geometry of the studied bladed disk. The element type is solid187 prismatic 10-nodes element that is commonly used for meshing of the imported geometry.

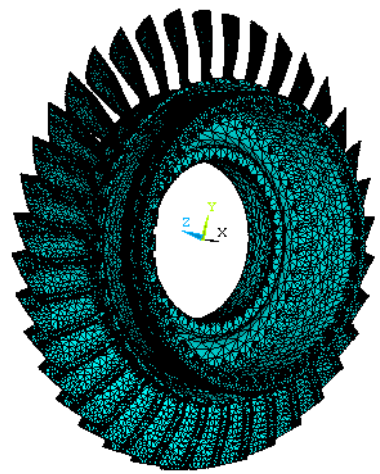

Fig. 19 Realistic finite elements model
Fig. 20 shows the simplified model of the bladed disk. Here the entire disk is modeled by solid185 8-nodes elements. The most controversial issue is the blade modeling. It was performed by shell181 4-nodes elements applying variable through blade height thickness. The blade profile is of rectangular shape. It has an essential influence on the model dynamic properties regarding the realistic model. The model geometrical and material properties are then adjusted in order to be closer to the characteristics of the realistic model.

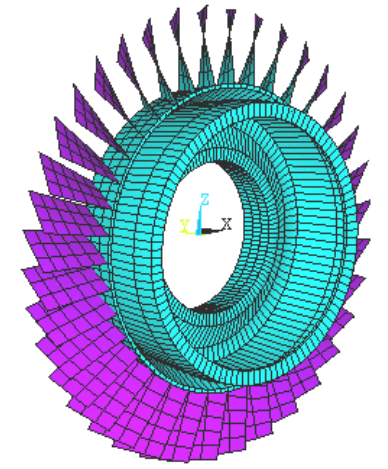

Fig. 20 Simplified finite elements model

Firstly, the modal analysis involving two models was accomplished at zero frequency of rotation in the conditions of cyclic symmetry. Also the question of the disk model restraining was considered. Fig. 19 and Fig. 20 correspond to the case of the disk without disk-support, which is used to adapt the bladed disk on the shaft of the test bench. Therefore the disk is clamped by the flank of rear cylindrical part (Fig. 21).

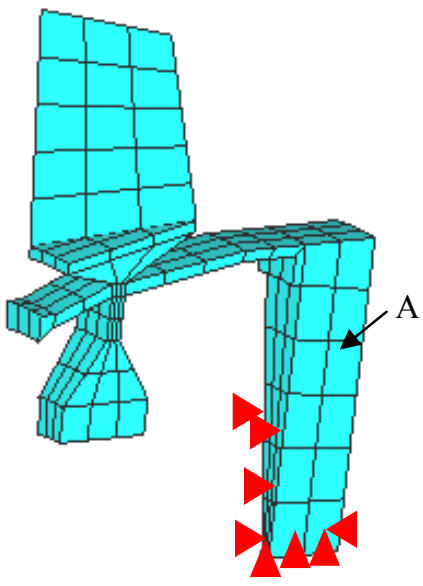

Fig. 21 Sector of simplified bladed disk model with supporting disk (A) and boundary conditions (Fig. 4)

The presented below frequency diagram of the bladed disk is constructed superposing results of simplified models from Fig. 20 and Fig. 21 and realistic model (Fig. 19). From the frequency diagram (Fig. 22) the approximation of the vibration characteristics is observed. It is of sufficiently enough level up to the family of modes associated with third blade (second flexural) mode. In the following simulation the simplified 
model with the supporting disk and electronic box will be used. It is noted from the diagram that adding of the disk support makes the structure less stiff lowering natural frequencies. Some modes such as 4ND-3 are practically not affected.

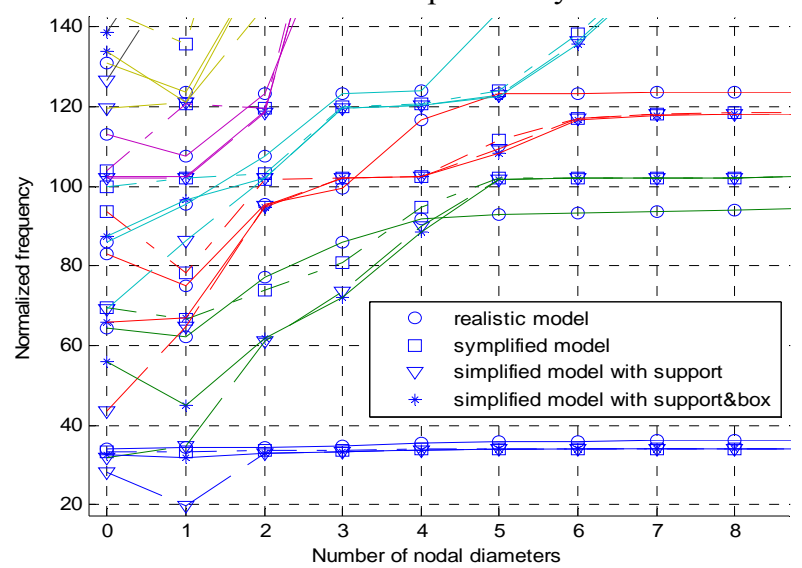

Fig. 22 Frequency diagram of the bladed disk

In the study we are interested in two modes, which will be analyzed and compared with the experimental data. They are second mode of two nodal diameters (2ND-2) and forth mode of four nodal diameters (4ND-4). The mode natural frequencies almost correspond to the experimental data, whereas in simulations it is not of the same order due to certain level of simplification.

Results of modal analysis of the simplified model show good correlation with the realistic model with exception of modes order. The mode 4ND-3 became 4ND-4 in the case of simplified model. It is mode associated with $1 \mathrm{~T}$ mode of blades. It can be explained by the fact that simplified disk is simulated as more stiff structure. Whereas in the case of realistic model we observe two close modes of four nodal diameters, which differ by the level of disk participation in the mode shapes. During experiments the mode 4ND-3 was chosen having higher level of the disk participation that made easier its excitation process by PZT. There is also mode 4ND-4, which of the same nature (associated with 1T mode of blades), but with much lower coupling between blades and disk.

Next we will consider the Coriolis effect influence by drawing the diagram of Campbell in the vicinity of the modes of interest.

\subsection{Coriolis Effect Influence on Bladed Disk Dynamics}

Coriolis forces effect should be considered before to pass to the forced response analysis of the blisk. As it was said earlier the effect of Coriolis forces is in most of cases neglected considering bladed disk dynamics. Generally, Coriolis forces are derived as components of blade and disk motion along the axes, which are perpendicular to the axis of disk rotation. At the same time the force components related with the disk motion in the parallel direction do not participate in the effect [12].

The analysis was performed in the rotating reference frame. The rotation speed was varied from 0 to $3100 \mathrm{rpm}$ that corresponds to the operational mode of the test bench (Fig. 1).
During simulation the stress-stiffening and spin-softening effects were not taken into account. The damped eigensolver was used for the problem solution. Results of simulation were compared with the test data obtained for the same mode type.

Experimental diagram of Campbell (Fig. 23) shows appearance of splitting of the mode corresponding resonance peak.

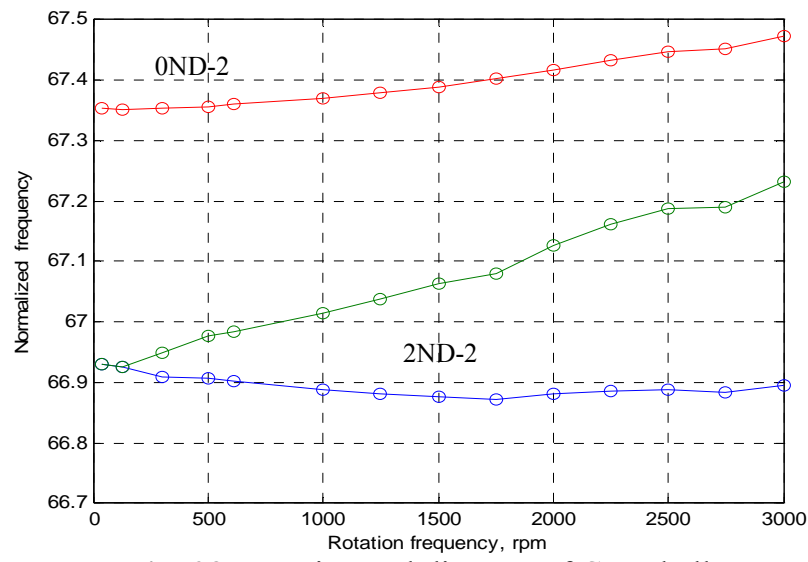

Fig. 23 Experimental diagram of Campbell: modes 2ND-2 and 0ND-2

The same modes were taken within the simplified model, with only difference that two modes are more distant in the frequency range (Fig. 24). It should be pointed out the low level of mistuning influence on the mode resonances from both experimental and simulation results. From the test data it is confirmed by the same resonance frequency at the zero rotation frequency. Concerning the implementation of mistuning to the model, this issue will be discussed in the next section. Here mistuned system is depicted by the dashed lines and the tuned one - by the solid lines.

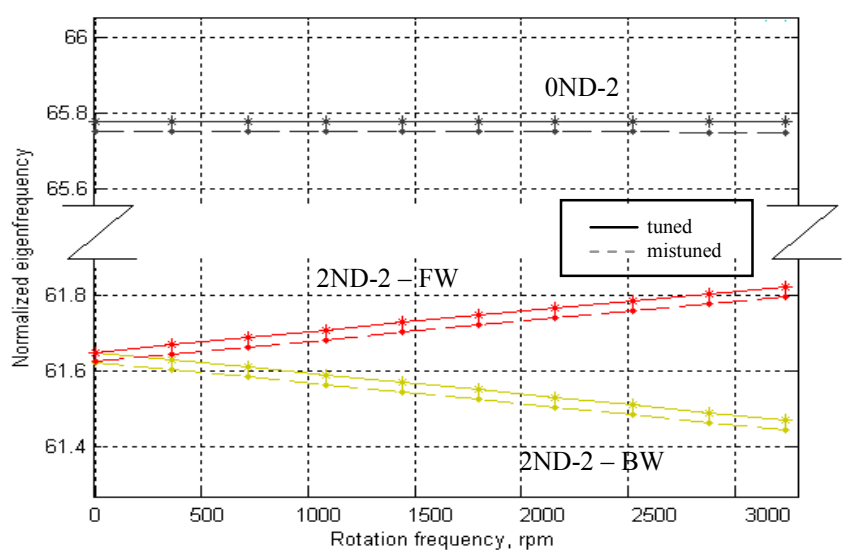

Fig. 24 Simulated diagram of Campbell: modes 2ND-2 and 0ND-2 (tuned/mistuned)

The second mode of interest is the mode of four nodal diameters (Fig. 25 and Fig. 26). As it was mentioned, in contrast to the nature, the order of the mode is not the same for the model and experimental data. 


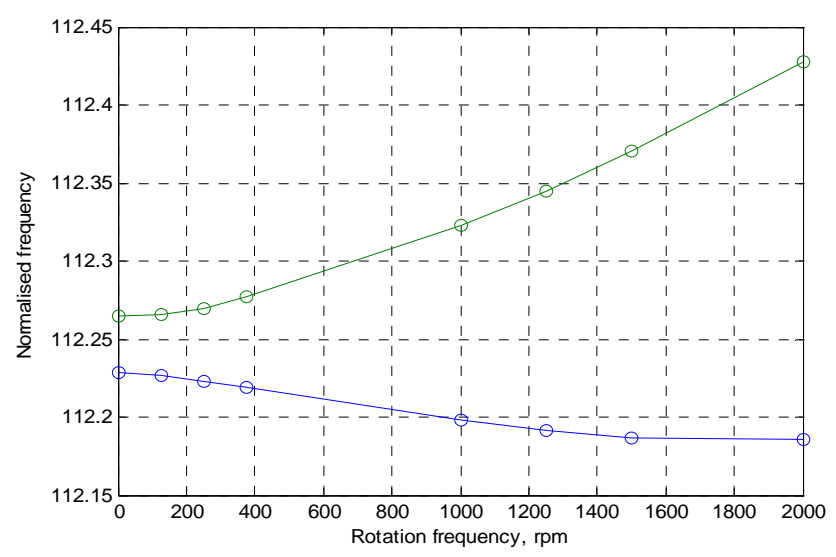

Fig. 25 Experimental diagram of Campbell: mode 4ND-3

From experimental results (Fig. 25) both effects of Coriolis and mistuning are observed. The last one is evident even at zero frequency of rotation making the resonance peak splitting of about $\sim 0.05$.

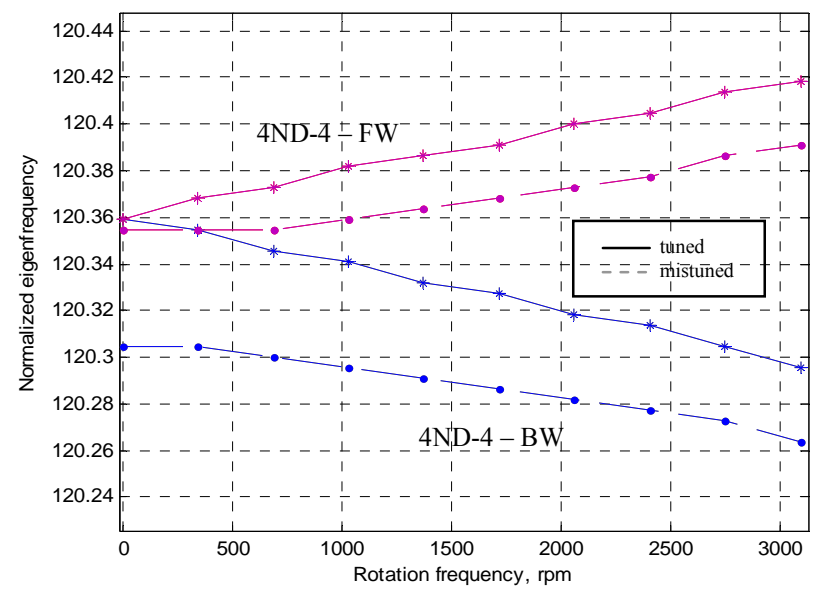

Fig. 26 Simulated diagram of Campbell: mode 4ND-4 (tuned/mistuned)

Comparing simulated data (Fig. 26) with the experiment (Fig. 25), the same relationships are depicted. But the level of simplification does not allow tolerating the equal proportions between mistuned and tuned cases and the resonance splitting value due to Coriolis effect. Though the simplified model confirms the effects revealed during experimental campaign stronger influence of mistuning and Coriolis forces.

\subsection{Forced Response of the Bladed Disk}

The most important issue regarding the forced response of the disk is the modeling of the excitation system. The realistic system is implemented using 24 piezoelectric actuators (PZT) grouped by 3 units (Fig. 2). The problem can be solved in two ways: (1) run coupled-field analysis using applicable elements to simulate PZT or (2) simplify PZT action by a pair of circumferentially spaced antagonist point forces as used in [4]. The last one was chosen as the most appropriate in the meaning of outcomes quality and computation time.
To excite the required modes the sign of excitation is varied depending on required nodal diameters number. In simulated excitation system 36 force pairs (Fig. 27) are grouped by their circumferential position. In this case the excitation is fixed regarding to the disk (standing wave excitation - sections 3.2 .4 , case 1 and 3.3 , case 3 ). It is described in experimental setup section that PZT circumferential distribution provides some spacing and it was shown that it prevents zero nodal diameters modes to respond (Fig. 6). It is especially prominent in the case of 4ND excitation. The same was provided within the model and it showed good accordance with experiments.

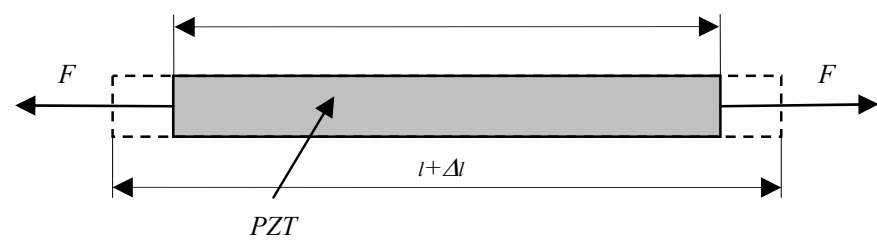

Fig. 27 Representative scheme of PZT excitation simulation

\subsection{Forced Response of Mistuned Bladed Disk}

Many authors admit that prediction of mistuning effect on the bladed disk response is a challenging problem [2], [13]. The difficulties are caused by two main complications:

- mistuning disrupts system cyclic symmetry, and

- mistuning leads to higher than expected amplitudes.

Mistuning effect on the bladed disk forced response can be represented by the stiffness mistuning. As far as we consider integrated bladed disk design the mistuning model can be represented by perturbation of the individual sector partition of the bladed disk stiffness matrix:

$$
\mathbf{K}_{j}^{s m}=\left(1+m_{j}\right) \mathbf{K}_{j}^{s},
$$

where $m_{j}$ is the mistuning coefficient of $j$-th sector randomly generated by normal law with mean zero and standard deviation $\sigma$.

In the following figures the responses of all blades are presented with solid lines corresponding to tuned system and dashed lines showing mistuned system response. The set level of mistuning is taken from experimental results and constitutes $0.4 \%$ or $\sigma=0.004$. The set level of damping is also obtained from the experimental campaign and constitutes the value of $0.035 \%$ for the $2 \mathrm{ND}-2$ mode, whereas the mode $4 \mathrm{ND}-4$ is less damped with $0.014 \%$ of damping.

Analyzing frequency response of the disk around the mode 2ND-2 (Fig. 28), influence of two phenomena is observed: Coriolis forces and mistuning. Unlike Campbell diagram the blisk's FRF of the mode at zero rotational frequency makes possible to observe the mistuning influence on the disk dynamics that is still remained very slight. 


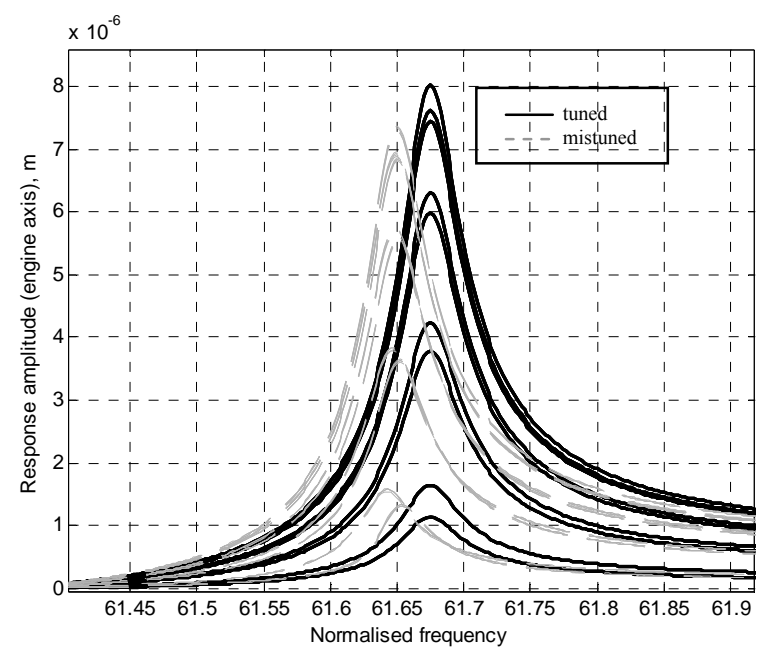

a

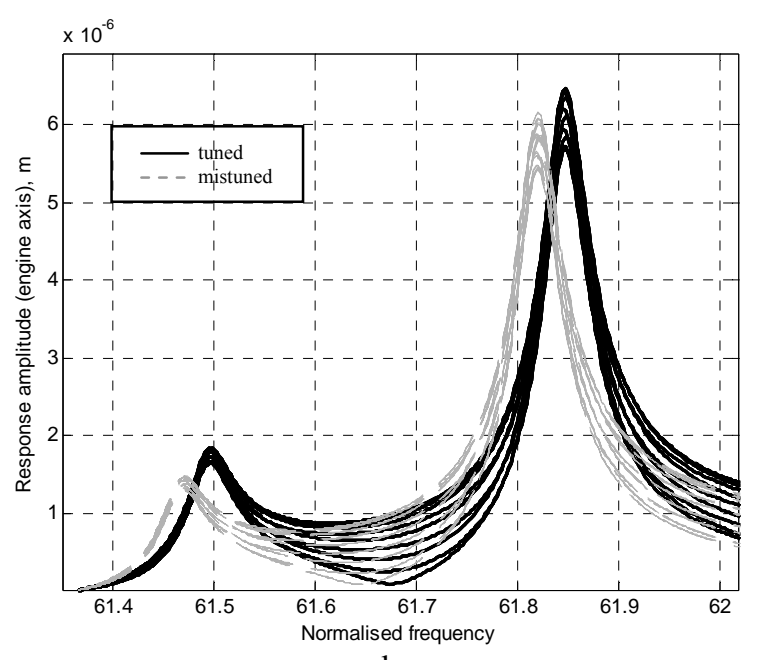

b

Fig. 28 Simulated FRF (all blades, mode $2 \mathrm{ND}-2$ ): $a-\Omega=0 \mathrm{rpm}, \mathrm{b}-\Omega=3100 \mathrm{rpm}$

Fig. 29 shows FRF of the bladed disk model for the mode 4ND-4. From the test results this mode is more subjected to the mistuning in the meaning of resonance peak shifting. At the same time the levels of splitting due to Coriolis effect of the resonance remain of almost same values for both tuned and mistuned systems (Fig. 26). At the zero rotational speed we do

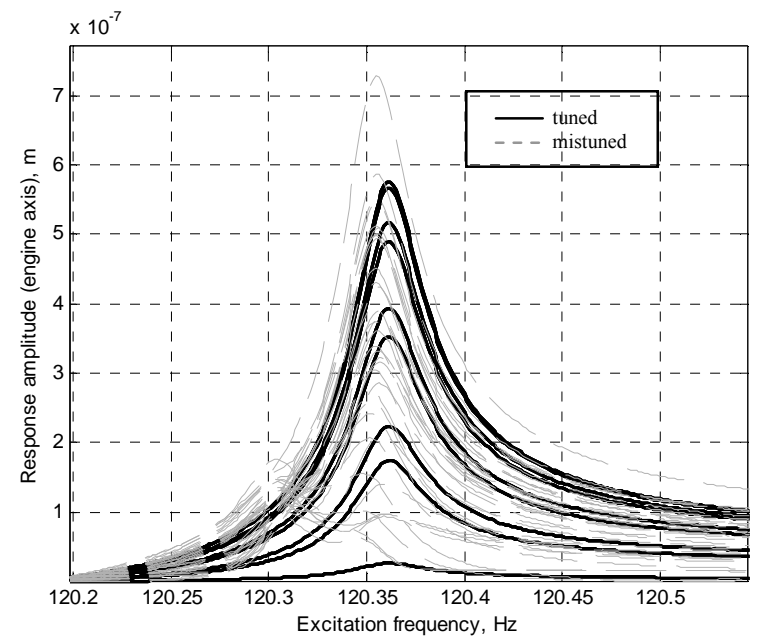

a not observe the resonance splitting neither for tuned nor mistuned cases (Fig. 29). The mistuning makes respond some other modes, but the main resonance is always forth mode of four nodal diameters. It should be noted the higher level of mistuning effect in comparison with the mode 2ND-2.

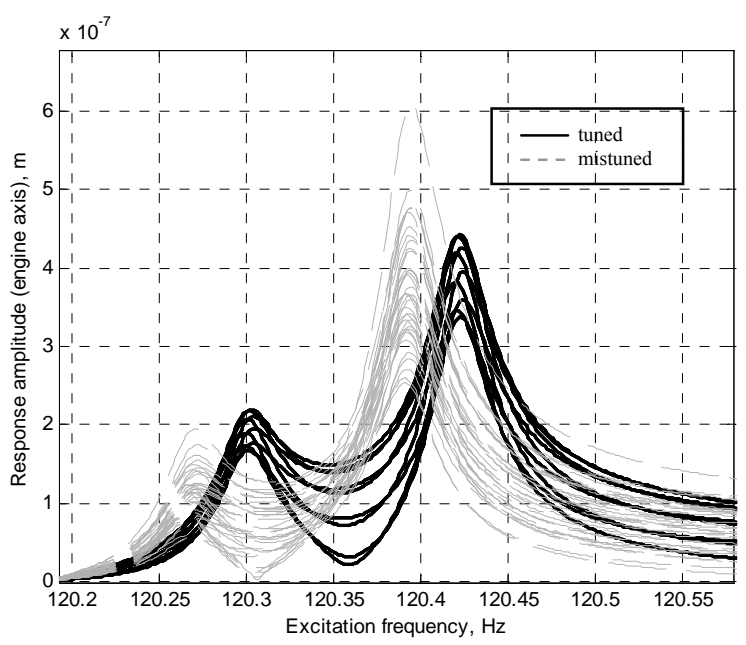

$\mathrm{b}$

Fig. 29 Simulated FRF (all blades, mode $4 \mathrm{ND}-4$ ): $a-\Omega=0 \mathrm{rpm}, \mathrm{b}-\Omega=3100 \mathrm{rpm}$

Besides overall mistuning influence on bladed disk response, it is revealed its unequal effect on resonances perturbation of forward and backward travelling waves. It is observed that forward branch is much more perturbed then the backward one.

As it was mentioned it is possible to see two resonance peaks corresponding to forward and backward travelling waves from the forced responses at the rotation speed $\Omega$ of both modes of interest. The phenomenon can be demonstrated tracing blade phase as a function of blade number (Fig. 30). The phase data are taken at the resonance frequencies of a blade with the highest response amplitude for both tuned and mistuned systems. The same effect of mode rotation in the case of standing wave excitation should be shown for mistuned system (Fig. 30b). It was also confirmed with the real blisk that present level of mistuning does not obstruct the modes rotation in the case of $\mathrm{FW}$ peak at frequency 120.42, whereas the response is almost standing for lower frequency peak. Observing phase profile of the mistuned lower frequency branch, we are approaching to shape of standing wave response as shown in experimental section (Fig. 13). The same can be derived from the amplitudes distribution of all blades (Fig. 31). 

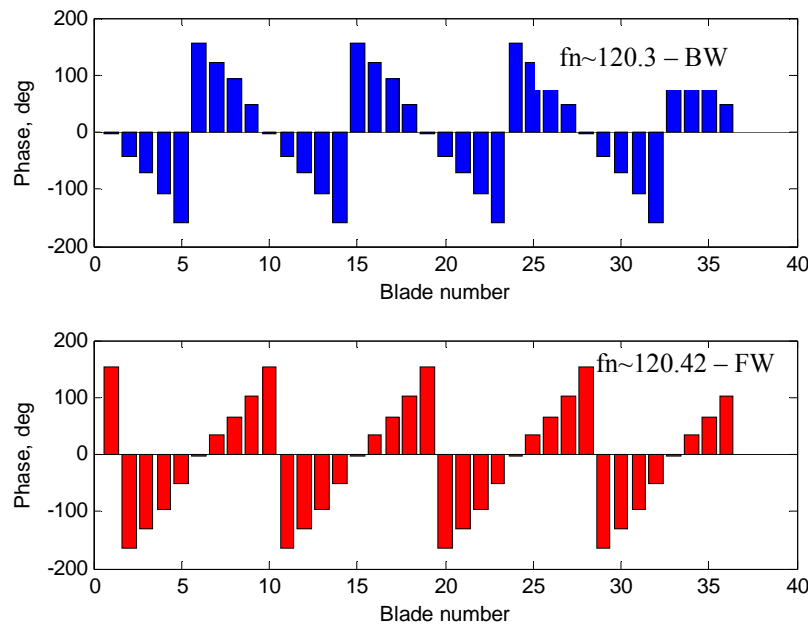
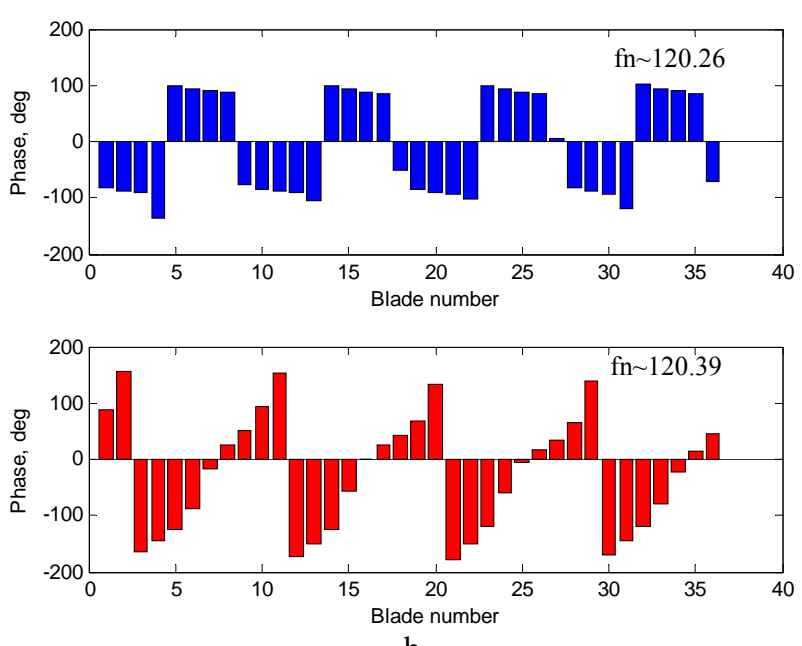

b

Fig. 30 Phases distribution of individual blades (mode 4ND-4, $3100 \mathrm{rpm}$ ): $\mathrm{a}-$ tuned system, $\mathrm{b}-$ mistuned system $(\sigma=0.004)$
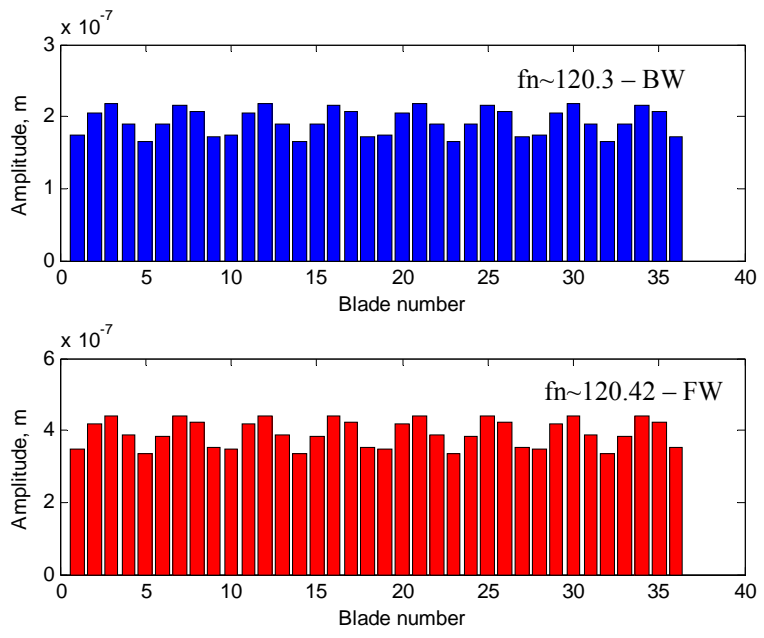

a
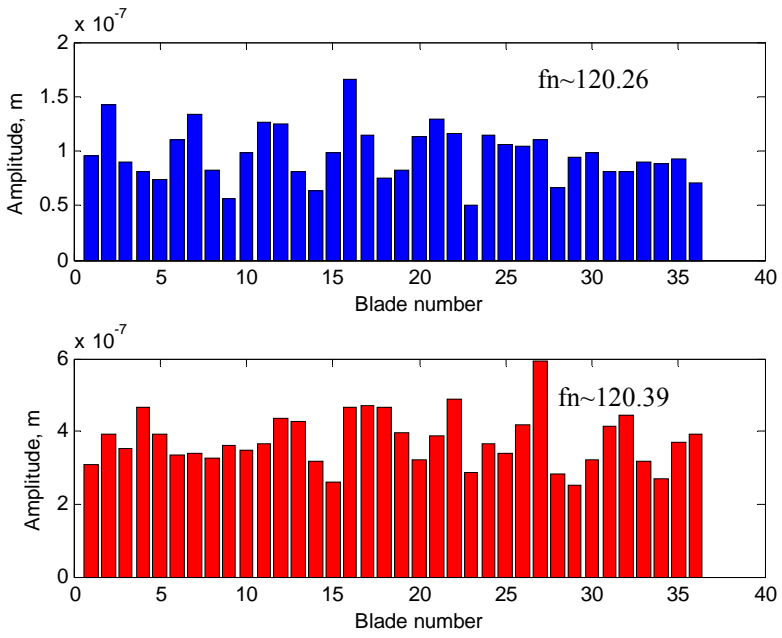

b

Fig. 31 Amplitudes distribution of individual blades (mode 4ND-4, $3100 \mathrm{rpm}$ ):

\section{CONCLUSIONS}

Coriolis effect has been shown to appear experimentally and numerically on the example of an actual design of high pressure compressor integrally bladed disk. An excitation system for generating rotating excitations on the rotating blisk has been presented and validated. The typical changes in the dynamic induced by Coriolis effect are shown and quantified experimentally and numerically. Modes affected by Coriolis effects are rather low nodal diameters coupled modes for which significant splits of frequencies appear with increase of the rotating speed. In cases where these modes are concerned by forced responses, it may be recommended to take into account the demonstrated effects for accurate prediction of bladed disks dynamic properties.

Finally, summarizing the study two main issues should be pointed out: (1) Coriolis effect on bladed disk dynamics and
(2) its common influence with mistuning on the blisk frequency response. The main conclusion made from the study is that Coriolis effect should be taken into account during early design stage of the bladed disks. It became more and more critical with modern tendencies of implementing of lighter and geometrically more complicated structures where the weight is saved thanking to the disk partition and blade thickness. In order to classify its influence two main signatures of Coriolis effect presence can be determined. Firstly, natural frequency change - splitting of a resonance peak that results in resonance appearing earlier or later with the same EO of excitation. In some cases it can lead to the mode to be excited by the not the same engine order as it was supposed. Secondly, it makes rotate a mode even in the case of standing wave excitation. This feature vanishes at the presence of mistuning (depending on its level [10]). Also, as shown experimentally, because of mistuning, a standing wave of response can appear at a 
resonance if the Coriolis effect is not present even if the excitation is rotating.

\section{ACKNOWLEDGMENTS}

This work is supported by Snecma, SAFRAN group within the frameworks of MAIA research technology program sponsored by CNRS and SAFRAN.

\section{REFERENCES}

[1]. ANSYS V12 Manual, 2009, ANSYS Inc

[2]. Bladh R., Castanier, M. P., Pierre, C., 2001, "Component-Mode-Based Reduced Order Modeling Techniques for Mistuned Bladed Disks - Part I: Theoretical models", ASME Journal of Engineering for Gas Turbines and Power, Vol. 123(1), pp. 100-108.

[3]. Charleux, D., 2006, "The study of friction effect at the blade foot on dynamics of the bladed disks", $\mathrm{PhD}$ thesis, Ecole Centrale de Lyon, France

[4]. Charleux D., Gibert C., Thouverez, F., Dupeux., J., 2006, "Numerical and experimental study of friction damping in blade attachments of rotating bladed disks". International Journal of Rotating Machinery, Vol. 2006, pp 1-13.

[5]. Huang B.W., Huang J.H., 2001, "Mode localization in a rotating mistuned turbodisk with Coriolis effect". International journal of mechanical sciences, Vol. 43(7), pp. 1643-1660.

[6]. Jacquet-Richardet, G., Ferraris, G., Rieutord, P., 1996, "Frequencies and modes of rotating flexible bladed disk-shaft assemblies: A global cyclic symmetry approach". Journal of sound and vibration, Vol. 191(5), pp. 901-915.

[7]. Jean P., Gibert C., Dupont C., Lombard J.-P., 2008, "Test-model correlation of dry-friction damping phenomena in aero-engines". Proceedings of ASME TurboExpo, 2008, GT2008-50891, Berlin, Germany

[8]. Jones, K., Cross, C., 2003, "Travelling wave excitation system for bladed disks". Journal of propulsion and power, Vol. 19 (1), pp. 134-141.

[9]. Judge, J., Pierre, C., Mehmed, O., 2001, "Experimental investigation of mode localisation and forced response amplitude magnification for a mistuned bladed disk". Transaction of ASME, Vol. 123, pp. 940-950.

[10]. Kharyton, V., Laine, J-P., Thouverez, F., Kucher, O. 2009, "Cracked blade detection from bladed disk forced response" ASME Paper GE2009-59598, Proceedings of the ASME Turboexpo2009, Orlando, FL, USA.

[11]. Laxalde, D., Gibert, C., Thouverez, F., 2008 "Experimental and Numerical Investigations of Friction Rings Damping of Blisks". Proceedings of ASME Turbo Expo, 2008, GT2008-50862, Berlin, Germany.

[12]. Nikolic, M., Petrov, E., Ewins, D. 2007, "Coriolis forces in forced response of mistuned bladed disks". Journal of turbomachinery, Vol. 129, pp. 730-739.

[13]. Pierre, C., Ceccio, S. L., Judge, J., Cross, C., 2000, "Experimental Investigation of Mistuned Bladed Disk Vibration". Proceedings of the 5th National Turbine Engine High Cycle Fatigue Conference, Chandler, Arizona, 2000.
[14]. Szwedowicz, J., Gibert, C. and al., 2006, "Numerical and experimental damping assessment of a thinwaled friction damper in the rotating set-up with high pressure turbine blades". Procedings of ASME TurboExpo 2006, GT 2006-90951, Barcelona, Spain. 